\title{
Twisted Plane Wave Expansions Using Hypercomplex Methods
}

\author{
by \\ Fabrizio Colombo, Irene Sabadini, Franciscus Sommen and \\ Daniele C. Struppa
}

\begin{abstract}
The purpose of this paper is to derive various representations of the Dirac delta distribution, including a Bony-type twisted Radon decomposition, from boundary values of monogenic functions. This leads to a new and simpler approach based on the properties of the analogue of the Cauchy kernel in the context of monogenic functions.
\end{abstract}

2010 Mathematics Subject Classification: Primary 30G35; Secondary 46F20.

Keywords: delta distribution, plane wave expansion, regular functions of a quaternionic variable, monogenic functions.

\section{$\S 1$. Introduction}

An important problem in the theory of distributions is the way in which distributions with very large microsupport can be written in terms of distributions each of which has a comparably smaller microsupport.

Maybe the first and clearest examples occurs for the Dirac delta $\delta$ in $\mathbb{R}$ which is supported at the origin, but whose microsupport contains both directions, namely it is $(0, \pm i)$. As is well known, one can represent the delta distribution as a sum of two distributions, each of which has a microsupport composed of a single point, i.e. $\delta=\delta^{+}+\delta^{-}$.

Communicated by H. Okamoto. Received March 6, 2013. Revised June 14, 2013.

F. Colombo: Politecnico di Milano, Dipartimento di Matematica, Via Bonardi 9, Milano, Italy; e-mail: fabrizio.colombo@polimi.it

I. Sabadini: Politecnico di Milano, Dipartimento di Matematica, Via Bonardi 9, Milano, Italy; e-mail: irene.sabadini@polimi.it

F. Sommen: Clifford Research Group, Faculty of Sciences, Ghent University,

Galglaan 2, 9000 Gent, Belgium;

e-mail: fs@cage.ugent.be

D. C. Struppa: Schmid College of Science and Technology, Chapman University,

Orange, CA 92866, USA;

e-mail: struppa@chapman.edu

(C) 2014 Research Institute for Mathematical Sciences, Kyoto University. All rights reserved. 
The same decomposition is true in the case of several variables, though now the sum is replaced by an integral over the unit sphere. This result is known as the Radon decomposition of the delta distribution.

The first goal of this paper is to show how Clifford analysis can be used to obtain this kind of results by exploiting the properties of the Cauchy kernels that are available in that context. In so doing, however, we will also discover new and different representations that are available because, for example, the delta distribution in $\mathbb{R}^{m}$ can be thought of as the sum of $2^{m}$ boundary values of holomorphic functions in $\mathbb{C}^{m}$, as well as the boundary values of two monogenic functions on $\mathbb{R}^{m+1}$.

We begin by reviewing the classical work of Kaneko and others on plane wave decomposition. We then consider the case of distributions in $\mathbb{R}^{3}$ as boundary values of the Cauchy kernel for regular functions on the space $\mathbb{H}$ of quaternions. We then extend the analysis to the higher dimensional case using monogenic functions and Clifford analysis.

The paper consists of three sections, besides the introduction. Section 2 deals with the various representations of the delta distribution, both in one and several variables. We also discuss how an idea inspired by the Fourier-Bros-Iagolnitzer transform leads to a representation of the Dirac delta by taking the real part of the restriction of the Cauchy kernel to a parabola, multiplied by a suitable factor, i.e.

$$
\delta(x)=2 \operatorname{Re}\left[-\left.\frac{1}{2 \pi i z}\right|_{y=a x^{2}}(1+a x)\right] .
$$

Section 3 treats the specific case of dimension 3 by making use of the representation of the so-called Cauchy-Fueter kernel and other techniques of quaternionic analysis. We generalize formula (1.1) to the three-dimensional case and, as a corollary, we derive a Bony-type formula. Finally, Section 4 generalizes this discussion to the case of dimension greater than 3 by using Clifford analysis techniques.

\section{§2. Representations of the delta distribution}

The delta distribution can be written in several ways. For example, it decomposes as the sum

$$
\delta(x)=\delta^{+}(x)+\delta^{-}(x)
$$

where

$$
\delta^{ \pm}(x)=\frac{1}{2}\left(\delta(x) \pm \frac{i}{\pi} \mathrm{PV} \frac{1}{x}\right)
$$


where PV denotes principal value. As a hyperfunction, the delta corresponds to

$$
\left[-\frac{1}{2 \pi i z}\right]
$$

We now use the Fourier-Bros-Iagolnitzer transform (see [2]) to represent the delta distribution. We recall (see [6, Lemma 3.33]) that

$$
\delta(x)=\int_{-\infty}^{\infty} e^{2 \pi\left(i x \xi-a x^{2}|\xi|\right)}(1+i a x \operatorname{sgn} \xi) d \xi, \quad a>0 .
$$

We use this result to show that the decomposition (2.1) holds.

Definition 2.1. Let $a$ be a positive real number. For every $f \in \mathcal{S}(\mathbb{R})$ we define the functionals

$$
\begin{aligned}
& \left\langle f, T_{+}\right\rangle:=\int_{-\infty}^{\infty} d x f(x) \int_{0}^{\infty} e^{2 \pi\left(i x-a x^{2}\right) \xi}(1+i a x) d \xi \\
& \left\langle f, T_{-}\right\rangle:=\int_{-\infty}^{\infty} d x f(x) \int_{-\infty}^{0} e^{2 \pi\left(i x+a x^{2}\right) \xi}(1-i a x) d \xi
\end{aligned}
$$

We have the following result (see [6]):

Theorem 2.2. Let $T_{ \pm}$be the functionals defined in (2.3) and (2.4). Then $T_{ \pm}$ define the distributions $T_{ \pm}=\delta^{ \pm}$.

Remark 2.3. An interesting consequence of the fact that our reasoning is carried out in the algebra of complex numbers, not in the real setting, is the following. From (2.2) it is immediate that the real part of $\delta^{+}$coincides with $\frac{1}{2} \delta$. Instead of considering $\delta(x)$ as the boundary value of $1 / 2 \pi i z$, we can restrict this function to the parabola $y=a x^{2}$ and obtain the equality

$$
\delta(x)=2 \operatorname{Re}\left[-\left.\frac{1}{2 \pi i z}\right|_{y=a x^{2}}(1+a x)\right] .
$$

This equality is crucial in this paper because we will show how it can be generalized in the Clifford algebra setting by using the notion of scalar part of a Clifford number (in particular, of a quaternion).

Let us now consider the case when $\underline{x}$ is a variable in $\mathbb{R}^{n}$, i.e. $\underline{x}=\left(x_{1}, \ldots, x_{n}\right)$; then the delta distribution is defined as

$$
\delta(\underline{x}):=\delta\left(x_{1}\right) \ldots \delta\left(x_{n}\right)=\prod_{j=1}^{n}-\frac{1}{2 \pi i}\left(\frac{1}{x_{j}+i 0}-\frac{1}{x_{j}-i 0}\right) .
$$


Let $\underline{\sigma}=\left(\sigma_{1}, \ldots, \sigma_{n}\right)$, where $\sigma_{j}= \pm 1$, let $\operatorname{sgn}(\underline{\sigma})=\sigma_{1} \ldots \sigma_{n}$, and define a cone, called the $\underline{\sigma}$-orthant, by

$$
\Gamma_{\underline{\sigma}}:=\left\{\underline{x} \in \mathbb{R}^{n}: x_{j} \sigma_{j}>0, j=1, \ldots, n\right\} .
$$

Then

$$
\delta(\underline{x})=\left(-\frac{1}{2 \pi i}\right)^{n} \sum_{\underline{\sigma}} \frac{\operatorname{sgn}(\underline{\sigma})}{\left(x_{1}+i \sigma_{1} 0\right) \ldots\left(x_{n}+i \sigma_{n} 0\right)} .
$$

Each summand can be interpreted as the boundary value on the wedge $\mathbb{R}^{n}+i \Gamma_{\underline{\sigma}}$ of the holomorphic function

$$
\frac{\operatorname{sgn}(\underline{\sigma})}{(-2 \pi i)^{n} z_{1} \ldots z_{n}} .
$$

More generally, any hyperfunction $f$ on $\Omega \subseteq \mathbb{R}^{n}$ can be written in the form

$$
f(\underline{x})=\sum_{j=1}^{N} F_{j}\left(\underline{x}+i \Gamma_{j} 0\right),
$$

where $\left\{F_{j}\right\}_{j=1}^{N}$ are holomorphic functions and each $F_{j}$ is defined on the infinitesimal wedge $\mathbb{R}^{n}+i \Gamma_{j} 0$. Let us recall that the singular support of a hyperfunction defined in a set $U$ is defined as the complement of the largest open subset on which the hyperfunction is real analytic. It is immediate that the singular support of $\delta$ is $\{0\}$.

Definition 2.4. Let $f$ be a hyperfunction defined on $\Omega \subseteq \mathbb{R}^{n}$. We say that $f$ is microanalytic at the point $\left(\underline{x}, \frac{1}{i} \xi d \underline{x} \infty\right)$, where $\underline{\xi}=\left(\xi_{1}, \ldots, \xi_{n}\right)$, if for a suitable boundary value representation of the form (2.6) of $f$ in a neighborhood of $\underline{x}$ we have

$$
\Gamma_{j} \cap\left\{\underline{y}=\left(y_{1}, \ldots, y_{n}\right) \in \mathbb{R}^{n}: \xi_{1} y_{1}+\ldots+\xi_{n} y_{n}>0\right\} \neq \emptyset \quad \forall j=1, \ldots, N .
$$

The set of points at which $f$ is not microanalytic is called the singular spectrum and it is denoted by S.S. $f$.

Remark 2.5. To simplify the notation we will write $\mp i \xi d \underline{x} \infty$ instead of $\pm(1 / i) \underline{\xi} d \underline{x} \infty$. Note that the singular spectrum is contained in a set that can be identified with $\mathbb{R}^{n} \times S^{n-1}$ where $S^{n-1}$ denotes the unit sphere centered at the origin in $\mathbb{R}^{n}$.

Remark 2.6. Let $\pi: \mathbb{R}^{n} \times S^{n-1} \rightarrow \mathbb{R}^{n}$ be the canonical projection. Then (see e.g. [9])

$$
\pi(\text { S.S. } f)=\operatorname{sing} \operatorname{supp} f \text {. }
$$

In the case of the delta distribution, S.S. $\delta=\{0\} \times S^{n-1}$. 
A different well known representation for $\delta$ can be formally obtained by the inverse Fourier transform

$$
\delta(x)=\frac{1}{(2 \pi)^{n}} \int_{\mathbb{R}^{n}} e^{i\langle\underline{x}, \underline{\xi}\rangle} d \underline{\xi}
$$

where $\langle\underline{x}, \underline{\xi}\rangle=x_{1} \xi_{1}+\cdots+x_{n} \xi_{n}$ is the usual inner product in $\mathbb{R}^{n}$. Then by considering polar coordinates $\xi=r \underline{\omega}, r \in \mathbb{R}^{+}, \underline{\omega} \in S^{n-1}$, we obtain

$$
\begin{aligned}
\delta(\underline{x}) & =\frac{1}{(2 \pi)^{n}} \int_{\mathbb{R}^{n}} e^{i\langle\underline{x}, \underline{\xi}\rangle} d \underline{\xi}=\frac{1}{(2 \pi)^{n}} \int_{S^{n-1}} d S(\underline{\omega}) \int_{0}^{\infty} e^{i\langle\underline{x}, \underline{\omega}\rangle r} r^{n-1} d r \\
& =\frac{(n-1) !}{(-2 \pi i)^{n}} \int_{S^{n-1}} \frac{d S(\underline{\omega})}{(\langle\underline{x}, \underline{\omega}\rangle+i 0)^{n}}
\end{aligned}
$$

where $d S(\underline{\omega})$ denotes the scalar element of surface area. This is usually referred to as the plane wave decomposition or Radon decomposition and its formally correct proof can be found e.g. in [8], [9]. Note that the singular spectrum of the integrand is $\{\langle\underline{x}, \underline{\omega}\rangle=0\}+i \underline{\omega}$. To obtain the decomposition in terms of objects for which the singular spectrum is just one point, one has to deform this Radon decomposition. This leads to the so-called Bony's twisted Radon decomposition (see e.g. [9, 4.2.10]):

$$
\delta(\underline{x})=\frac{(n-1) !}{(-2 \pi i)^{n}} \int_{S^{n-1}} \frac{1+i\langle\underline{x}, \underline{\omega}\rangle}{\left(\langle\underline{x}, \underline{\omega}\rangle+i|\underline{x}|^{2}+i 0\right)^{n}} d S(\underline{\omega}) .
$$

We will show how this decomposition can be easily obtained in the framework of quaternionic and Clifford analysis (see Corollaries 3.11 and 4.9).

\section{§3. The delta distribution in three dimensions}

Let us now consider the delta distribution in the three-dimensional space by identifying $\mathbb{R}^{3}$ with the set of purely imaginary quaternions. So, from now on, we will work in the real associative algebra $\mathbb{H}$ of quaternions with the standard basis $1, i$, $j, k$ such that

$$
i^{2}=j^{2}=k^{2}=-1, \quad i j=-j i=k, \quad j k=-k j=i, \quad k i=-i k=j .
$$

We write an element $q \in \mathbb{H}$ in the form

$$
q=x_{0}+i x_{1}+j x_{2}+k x_{3}
$$

where $x_{\ell} \in \mathbb{R}$ for $\ell=0,1,2,3$, and we set

$$
\operatorname{Re} q=x_{0}, \quad \operatorname{Im} q=\underline{q}=i x_{1}+j x_{2}+k x_{3}, \quad|q|=\sqrt{x_{0}^{2}+x_{1}^{2}+x_{2}^{2}+x_{3}^{2}}
$$


$\operatorname{Re} q, \operatorname{Im} q$ and $|q|$ are called the real (or scalar) part, the imaginary part and the modulus of $q$, respectively. The conjugate of a quaternion $q$ is $\bar{q}=\operatorname{Re} q-\operatorname{Im} q=$ $x_{0}-i x_{1}-j x_{2}-k x_{3}$. We will consider the so-called Cauchy-Fueter operator

$$
\frac{\partial_{l}}{\partial \bar{q}}=\frac{\partial}{\partial x_{0}}+i \frac{\partial}{\partial x_{1}}+j \frac{\partial}{\partial x_{2}}+k \frac{\partial}{\partial x_{3}} ;
$$

we say that a real differentiable function $f: U \rightarrow \mathbb{H}$, where $U \subseteq \mathbb{H}$ is an open set, is Cauchy-Fueter regular if it is in the kernel of the Cauchy-Fueter operator. We refer the reader to [3] and [14] for further details. In [5], [10] (see also [11], [12] for a more general case) it is shown how to develop a hyperfunction theory on the space $\widetilde{\mathbb{H}}$ of purely imaginary quaternions in complete analogy with the theory of classical hyperfunctions in one real variable. In this case, in particular, hyperfunctions on $\widetilde{\mathbb{H}}$ are defined as sums of boundary values of functions which are regular on $\mathbb{H} \backslash \widetilde{\mathbb{H}}$. Therefore one can expect that the arguments that we have just illustrated in the real case should also hold true in the case of $\widetilde{\mathbb{H}}$.

Let us recall that the analogue of the Cauchy kernel for functions regular in the sense of Cauchy-Fueter is the function

$$
G(q)=\frac{1}{2 \pi^{2}} \frac{\bar{q}}{|q|^{4}}
$$

We now prove the following result:

Theorem 3.1. Let $S^{2}$ be the sphere of unit, purely imaginary quaternions and let $x_{0} \neq 0$. Then

$$
G(q)=\frac{1}{(2 \pi)^{3}} \int_{S^{2}}\left(\langle\underline{q}, \underline{\omega}\rangle-x_{0} \underline{\omega}\right)^{-3} \underline{\omega} d S(\underline{\omega})
$$

where $d S(\underline{\omega})$ denotes the scalar element of surface area.

Proof. First of all we note that both sides are regular functions of axial type (see [1]), thus it is sufficient to verify the formula by taking $\underline{q}=0$. The result follows immediately.

Let us recall that, as hyperfunctions on $\widetilde{\mathbb{H}}$,

$$
\delta(\underline{q})=\mathrm{BV}\left[\frac{1}{2 \pi^{2}} \frac{\bar{q}}{|q|^{4}}\right],
$$

where BV stands for boundary value, and therefore

$$
\delta(\underline{q})=\frac{1}{(2 \pi)^{3}} \int_{S^{2}} \operatorname{BV}\left[\left(\langle\underline{q}, \underline{\omega}\rangle-x_{0} \underline{\omega}\right)^{-3} \underline{\omega}\right] d S(\underline{\omega}) .
$$


In addition (see e.g. [12])

$$
\delta^{+}(\underline{q})=\frac{1}{2} \delta(\underline{q})-\frac{1}{2 \pi^{2}} \frac{\underline{q}}{|q|^{4}},
$$

from which it follows that the scalar part of $2 \delta^{+}$gives $\delta$.

As is well known, in the sense of hyperfunctions,

$$
\delta^{\prime \prime}(x)=-\frac{1}{\pi i} \mathrm{BV}\left[\frac{1}{z^{3}}\right],
$$

and by formally replacing in $z=x+i y$ : $x$ by $\langle\underline{q}, \underline{\omega}\rangle, y$ by $x_{0}$ and $i$ by $-\underline{\omega}$, we obtain (see e.g. [7])

$$
\delta(\underline{q})=\frac{1}{2 \pi^{2}} \int_{S^{2}} \delta^{\prime \prime}(\langle\underline{q}, \underline{\omega}\rangle) d S(\underline{\omega})
$$

which expresses the Dirac delta over 3-dimensional space by means of the derivative of the one-dimensional delta. There exists a more refined Radon decomposition of the delta for which we first prove an alternative form of the Radon decomposition of the Cauchy kernel in Clifford analysis. We also have the following result:

Proposition 3.2. If $x_{0} \neq 0$, then

$$
G(q)=-i \frac{1}{(2 \pi)^{3}} \int_{S^{2}} \frac{1+i \underline{\omega}}{\left(\langle\underline{q}, \underline{\omega}\rangle+i x_{0}\right)^{3}} d S(\underline{\omega}) .
$$

Proof. Let us consider the two mutually annihilating idempotents

$$
\Omega_{ \pm}:=\frac{1}{2}(1 \pm i \underline{\omega}), \quad \underline{\omega} \in S^{2},
$$

and observe that

$$
1=\Omega_{+}+\Omega_{-} .
$$

Simple computations show that

$$
\underline{\omega} \Omega_{ \pm}=\mp i \Omega_{ \pm} .
$$

Let us rewrite the integrand in (3.1) as

$$
\frac{\Omega_{+}+\Omega_{-}}{\left(\langle\underline{x}, \underline{\omega}\rangle-x_{0} \underline{\omega}\right)^{3}} \underline{\omega}
$$

we have

$$
\begin{aligned}
\frac{\Omega_{+}}{\left(\langle\underline{x}, \underline{\omega}\rangle-x_{0} \underline{\omega}\right)^{3}} \underline{\omega} & =\frac{\Omega_{+}\left(\langle\underline{x}, \underline{\omega}\rangle+x_{0} \underline{\omega}\right)^{3}}{\left(\langle\underline{x}, \underline{\omega}\rangle^{2}+x_{0}^{2}\right)^{3}} \underline{\omega}=\frac{\left(\langle\underline{x}, \underline{\omega}\rangle-i x_{0}\right)^{3}(-i) \Omega_{+}}{\left(\langle\underline{x}, \underline{\omega}\rangle^{2}+x_{0}^{2}\right)^{3}} \\
& =-i \frac{\Omega_{+}}{\left(\langle\underline{x}, \underline{\omega}\rangle+i x_{0}\right)^{3}}
\end{aligned}
$$


and similarly

$$
\frac{\Omega_{-}}{\left(\langle\underline{x}, \underline{\omega}\rangle-x_{0} \underline{\omega}\right)^{3}} \underline{\omega}=i \frac{\Omega_{-}}{\left(\langle\underline{x}, \underline{\omega}\rangle-i x_{0}\right)^{3}} .
$$

Thus, from (3.1), we have

$$
\begin{aligned}
G(q) & =\frac{1}{(2 \pi)^{3}} \int_{S^{2}}\left(\langle\underline{q}, \underline{\omega}\rangle-x_{0} \underline{\omega}\right)^{-3} \underline{\omega} d S(\underline{\omega}) \\
& =\frac{1}{(2 \pi)^{3}} \int_{S^{2}} \frac{(-i) \Omega_{+}}{\left(\langle\underline{x}, \underline{\omega}\rangle+i x_{0}\right)^{3}} d S(\underline{\omega})+\frac{1}{(2 \pi)^{3}} \int_{S^{2}} \frac{(+i) \Omega_{-}}{\left(\langle\underline{x}, \underline{\omega}\rangle-i x_{0}\right)^{3}} d S(\underline{\omega}) \\
& =\frac{1}{(2 \pi)^{3}} \int_{S^{2}} \frac{(-i) \Omega_{+}}{\left(\langle\underline{x}, \underline{\omega}\rangle+i x_{0}\right)^{3}} d S(\underline{\omega})+\frac{1}{(2 \pi)^{3}} \int_{S^{2}} \frac{(-i) \Omega_{+}}{\left(\langle\underline{x}, \underline{\omega}\rangle+i x_{0}\right)^{3}} d S(\underline{\omega}),
\end{aligned}
$$

from which the desired equality follows.

Remark 3.3. This result is a refinement of (3.3) in the sense that in (3.3) the singular spectra of the delta (which is the entire $\{0\} \times S^{2}$ ) and of the integrand are the same. Here the singularities belong to the plane $\langle\underline{q}, \underline{\omega}\rangle=0$ in the direction of $\underline{\omega}$ only.

We are now ready to prove the quaternionic version of the planar Radon decomposition (2.7), which will allow us to write the delta as the integral of distributions each of which has singular support in a specific direction of $S^{2}$.

Theorem 3.4. The delta distribution on $\widetilde{\mathbb{H}}$ can be represented as

$$
\delta(\underline{q})=-i \frac{1}{(2 \pi)^{3}} \int_{S^{2}} \frac{1}{\left(\langle\underline{q}, \underline{\omega}\rangle+i 0^{+}\right)^{3}} d S(\underline{\omega}) .
$$

Proof. By taking the limit of (3.4) as $x_{0} \rightarrow 0^{+}$we obtain the new formula

$$
\delta^{+}(\underline{q})=-i \frac{1}{(2 \pi)^{3}} \int_{S^{2}} \frac{1+i \underline{\omega}}{(\langle\underline{q}, \underline{\omega}\rangle+i 0)^{3}} d S(\underline{\omega})
$$

whose scalar part (in the sense of the complexified algebra of quaternions $\mathbb{H}_{\mathbb{C}}$ ) gives the delta distribution on $\widetilde{\mathbb{H}}$ as in the statement.

Remark 3.5. Note that the singular spectrum of

$$
\frac{1}{(\langle\underline{q}, \underline{\omega}\rangle+i 0)^{3}}
$$

is $\{\underline{q}+i \underline{\omega}:\langle\underline{q}, \underline{\omega}\rangle=0\}$. This is the analog of the decomposition of the delta as $\delta^{+}+\delta^{-}$in $\mathbb{R}$. In fact the singular spectrum of the delta is $\{0\} \times S^{0}$ while $\delta^{ \pm}$have singular spectra $0 \pm i$. 
Remark 3.6. Until now we have looked at the delta as the boundary value of a function which is Cauchy-Fueter regular in $\mathbb{H} \backslash \widetilde{\mathbb{H}}$. On the other hand, the $\delta$ at the origin of $\mathbb{R}^{3}$ can also be considered as a suitable sum of boundary values of holomorphic functions in cones in $\mathbb{C}^{3}$. Specifically, we can go back to the planar Radon decomposition for the $\delta$ in $\mathbb{R}^{3}$,

$$
\delta(\underline{q})=-i \frac{1}{2 \pi^{2}} \int_{S^{2}} \frac{1}{\left(\langle\underline{q}, \underline{\omega}\rangle+i 0^{+}\right)^{3}} d S(\underline{\omega}),
$$

and note that $\langle\underline{q}, \underline{\omega}\rangle+i 0$ can be rewritten as $\langle\underline{q}+i \underline{p}, \omega\rangle$ with $\langle\underline{p}, \underline{\omega}\rangle>0$ and $\underline{p} \rightarrow \underline{0}$. Thus the integrand in the Radon decomposition can be seen as the boundary value of

$$
\frac{1}{(\langle\underline{q}, \underline{\omega}\rangle+i 0)^{3}}=\lim _{\langle\underline{p}, \underline{\omega}\rangle>0,\langle\underline{p}, \underline{\omega}\rangle \rightarrow 0} \frac{1}{(\langle\underline{q}+i \underline{p}, \underline{\omega}\rangle)^{3}}=\left.\lim _{\underline{p} \rightarrow 0} \mathrm{BV} \frac{1}{\langle\underline{z}, \underline{\omega}\rangle}\right|_{\langle\underline{p}, \underline{\omega}\rangle>0},
$$

where $\underline{z}=\underline{q}+i \underline{p}$ is an element of $\widetilde{\mathbb{H}}_{\mathbb{C}}$.

Notation. The scalar part of a quaternion $q$ will be denoted by $[q]_{0}$.

Proposition 3.7. The delta distribution can be written in the form

$$
\delta(\underline{q})=\frac{1}{2 \pi^{2}}\left[\int_{\widetilde{\mathbb{H}}} e^{i\langle\underline{q}, \underline{\xi}\rangle-x_{0}|\underline{\xi}|}\left(1+i \frac{\underline{\xi}}{|\underline{\xi}|}\right) d \xi_{1} d \xi_{2} d \xi_{3}(1-a \underline{q})\right]_{0} .
$$

Proof. To prove this result, we need to deform the contour of integration and instead of looking at the delta as

$$
\delta(\underline{q})=\frac{1}{(2 \pi)^{3}} \int_{\mathbb{R}^{3}} e^{i\langle\underline{q}, \underline{\xi}\rangle} d \xi_{1} d \xi_{2} d \xi_{3},
$$

we can consider the integral over the curve $\gamma(\underline{\xi})=\underline{\xi}+i a \underline{q} \mid \underline{\xi}$. We thus rewrite the integral by replacing $e^{i\langle\underline{q}, \underline{\xi}\rangle}$ with

$$
e^{i\langle\underline{q}, \underline{\xi}+i a \underline{q}|\underline{\xi}|\rangle}=e^{i\langle\underline{q}, \underline{\xi}\rangle-a|\underline{q}|^{2}|\underline{\xi}|} ;
$$

the volume form $V(d \underline{\xi}):=d \xi_{1} d \xi_{2} d \xi_{3}$ becomes

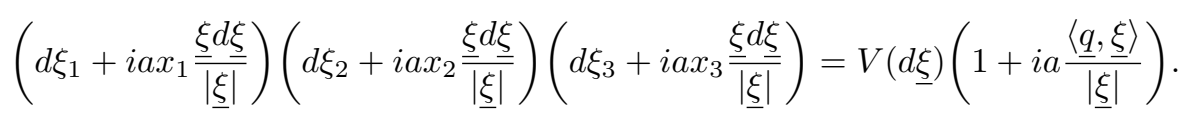

We now use the following identity (which can be easily verified by direct computations):

$$
1+i a \frac{\langle\underline{q}, \underline{\xi}\rangle}{|\underline{\xi}|}=\left[\left(1+i \frac{\underline{\xi}}{|\underline{\xi}|}\right)(1-a \underline{q})\right]_{0} .
$$

By setting $x_{0}=a|\underline{q}|^{2}$ we see that $\delta(\underline{q})$ can be written in the form (3.5). 
Remark 3.8. Similarly, when $x_{0}=a|x|^{2}$ we obtain a Fourier type integral with a monogenic exponential:

$$
2 G(q)=\frac{1}{2 \pi^{2}} \int_{\widetilde{\mathbb{H}}} e^{i\langle q, \xi\rangle-x_{0}|\underline{\xi}|}\left(1+i \frac{\underline{\xi}}{|\underline{\xi}|}\right) d \xi_{1} d \xi_{2} d \xi_{3},
$$

without using the Radon transform.

This leads to the following result, analogous to (2.5) and (3.5), which will be proved using quaternionic analysis:

Theorem 3.9. Let $x_{0}=a|\underline{q}|^{2}$. Then

$$
\delta(\underline{q})=\frac{1}{2 \pi^{2}}\left[\frac{x_{0}-\underline{q}}{\left|x_{0}+\underline{q}\right|^{4}}(1-a \underline{q})\right]_{0}=\frac{1}{2 \pi^{2}}\left[\frac{a|\underline{q}|^{2}-\underline{q}}{\left.|a| \underline{q}\right|^{2}+\left.\underline{q}\right|^{4}}(1-a \underline{q})\right]_{0}
$$

To prove this result we need the following lemma which, for simplicity, will be proved for the paraboloid $x_{0}=a|q|^{2}$ with $a=1$. The result can be proven with a similar technique also for every $a>0$.

Lemma 3.10. Let $f$ be a Fueter regular function for $x_{0}>0$. Then

$$
\left.\partial_{x_{0}} f\left(x_{0}+\underline{q}\right)\right|_{x_{0}=|\underline{q}|^{2}}=-\frac{2 \underline{q}+1}{4|\underline{q}|^{2}+1} \partial_{q} f\left(|\underline{q}|^{2}+\underline{q}\right) .
$$

Proof. We will write $\dot{\partial}_{r} f\left(x_{0}, \dot{q}\right)$ to indicate that the derivative $\dot{\partial}_{r}$ acts only on the variable $\underline{\dot{q}}$. Setting $\underline{q}=r \underline{\omega}$ we have

$$
\partial_{r} f\left(r^{2}, \underline{q}\right)=\left.2 r \partial_{x_{0}} f\left(x_{0}, \underline{q}\right)\right|_{x_{0}=r^{2}}+\dot{\partial}_{r} f\left(x_{0}, \underline{\dot{q}}\right) .
$$

For Fueter regular functions we also have

$$
\dot{\partial}_{\underline{q}} f\left(r^{2}, \underline{\dot{q}}\right)=\underline{\omega}\left(\dot{\partial}_{r}+\frac{1}{r} \dot{\Gamma}_{\underline{\omega}}\right) f\left(r^{2}, \underline{\dot{q}}\right)=-\left.\partial_{x_{0}} f\left(x_{0}, \underline{q}\right)\right|_{x_{0}=r^{2}} .
$$

Since $\dot{\Gamma}_{\underline{\omega}} f\left(r^{2}, \underline{\dot{q}}\right)=\Gamma_{\underline{\omega}} f\left(r^{2}, \underline{q}\right)$ we have

$$
\dot{\partial}_{\underline{q}} f\left(r^{2}, \underline{\dot{q}}\right)=\left.\underline{\omega} \partial_{x_{0}} f\left(x_{0}, \underline{q}\right)\right|_{x_{0}=r^{2}}-\frac{1}{r} \Gamma_{\underline{\omega}} f\left(r^{2}, \underline{q}\right)
$$

and we get

$$
\left(\partial_{r}+\frac{1}{r} \Gamma_{\underline{\omega}}\right) f\left(r^{2}, r \underline{\omega}\right)=\left.(2 r+\underline{\omega}) \partial_{x_{0}} f\left(x_{0}, r \underline{\omega}\right)\right|_{x_{0}=r^{2}},
$$

so finally

$$
(2 \underline{q}-1)^{-1} \partial_{\underline{q}} f\left(r^{2}, r \underline{\omega}\right)=\left.\partial_{x_{0}} f\left(x_{0}, r \underline{\omega}\right)\right|_{x_{0}=r^{2}} .
$$


Proof of Theorem 3.9. In order to prove the statement we put for simplicity $a=1$, and we compute the integral

$$
\int_{\mathbb{R}^{3}} \frac{1}{2 \pi^{2}}\left[\frac{|\underline{q}|^{2}-\underline{q}}{\left.|| \underline{q}\right|^{2}+\left.\underline{q}\right|^{4}}(1-\underline{q})\right]_{0} d x_{1} d x_{2} d x_{3} .
$$

Denote by $B(\varepsilon)$ the ball with center at the origin and radius $\varepsilon$; then the integral equals

$$
\begin{aligned}
\int_{\mathbb{R}^{3} \backslash B(\varepsilon)} \frac{1}{2 \pi^{2}}\left[\frac{|\underline{q}|^{2}-\underline{q}}{\left.|| \underline{q}\right|^{2}+\left.\underline{q}\right|^{4}}(1-\underline{q})\right]_{0} d x_{1} d x_{2} d x_{3} \\
+\int_{B(\varepsilon)} \frac{1}{2 \pi^{2}} \frac{|\underline{q}|^{2}-\underline{q}}{\left.|| \underline{q}\right|^{2}+\left.\underline{q}\right|^{4}}(1-\underline{q}) d x_{1} d x_{2} d x_{3}
\end{aligned}
$$

since the integral over $B(\varepsilon)$ of the nonscalar part vanishes. Now observe that also the integral over $\mathbb{R}^{3} \backslash B(\varepsilon)$ vanishes, since

$$
\frac{|-\underline{q}|^{2}+\underline{q}}{||-\left.\underline{q}\right|^{2}-\left.\underline{q}\right|^{4}}(1+\underline{q})=\frac{\underline{q}(1-\underline{q})}{\left.|| \underline{q}\right|^{2}-\left.\underline{q}\right|^{4}}(1+\underline{q})=-\frac{|\underline{q}|^{2}-\underline{q}}{\left.|| \underline{q}\right|^{2}+\left.\underline{q}\right|^{4}}(1-\underline{q})
$$

thus it is sufficient to compute

$$
\int_{B(\varepsilon)} \frac{1}{2 \pi^{2}} \frac{|\underline{q}|^{2}-\underline{q}}{\left.|| \underline{q}\right|^{2}+\left.\underline{q}\right|^{4}}(1-\underline{q}) d x_{1} d x_{2} d x_{3} .
$$

Let us now set

$$
\Lambda\left(x_{0}, \underline{q}\right)=-\frac{1}{\pi^{2}} \int_{0}^{\infty} \frac{\left(x_{0}+s\right)-\underline{q}}{\left|\left(x_{0}+s\right)+\underline{q}\right|^{4}} d s .
$$

The function $\Lambda\left(x_{0}, \underline{q}\right)$ is regular for $x_{0}>0$ and $\Lambda(0, \underline{q}) \in \mathcal{L}_{1}^{\text {loc }}\left(\mathbb{R}^{3}\right)$. Its derivative with respect to $x_{0}$ is

$$
\partial_{x_{0}} \Lambda\left(x_{0}, \underline{q}\right)=\frac{1}{\pi^{2}} \frac{x_{0}-\underline{q}}{\left|x_{0}+\underline{q}\right|^{4}} .
$$

Let us set $|\underline{q}|=r$ and, using Lemma 3.10, let us write the integrand in (3.8) as

$$
\frac{1}{\pi^{2}} \frac{r^{2}-\underline{q}}{\left|r^{2}-\underline{x}\right|^{4}}(1-\underline{q})=-\frac{(2 \underline{q}+1)(1-\underline{q})}{4 r^{2}+1} \partial_{\underline{q}} \Lambda(\underline{q})
$$


denoting by $H$ the Heaviside function, the integral (3.8) equals

$$
\begin{gathered}
-\lim _{\varepsilon \rightarrow 0} \int_{\mathbb{R}^{3}} \frac{1+2 r^{2}+\underline{q}}{4 r^{2}+1} H\left(\varepsilon^{2}-r^{2}\right) \Lambda(\underline{q}) d x_{1} d x_{2} d x_{3} \\
=\lim _{\varepsilon \rightarrow 0}\left(\int_{\mathbb{R}^{3}} \partial_{\underline{q}}\left(H\left(\varepsilon^{2}-r^{2}\right)\right) \frac{1+2 r^{2}+\underline{q}}{4 r^{2}+1} \Lambda(\underline{q}) d x_{1} d x_{2} d x_{3}\right. \\
\left.\quad+\int_{\mathbb{R}^{3}} H\left(\varepsilon^{2}-r^{2}\right) \partial_{\underline{q}}\left(\frac{1+2 r^{2}+\underline{q}}{4 r^{2}+1}\right) \Lambda(\underline{q}) d x_{1} d x_{2} d x_{3}\right) \\
=\lim _{\varepsilon \rightarrow 0}\left(\int_{\mathbb{R}^{3}} \partial_{\underline{x}}\left(H\left(\varepsilon^{2}-r^{2}\right)\right) \frac{1+2 r^{2}+\underline{q}}{4 r^{2}+1} \Lambda(\underline{q}) d x_{1} d x_{2} d x_{3}\right. \\
\left.\quad+\int_{B(\varepsilon)} \partial_{\underline{q}}\left(\frac{1+2 r^{2}+\underline{q}}{4 r^{2}+1}\right) \Lambda(\underline{q}) d x_{1} d x_{2} d x_{3}\right) .
\end{gathered}
$$

We have

$$
\lim _{\varepsilon \rightarrow 0} \int_{B(\varepsilon)} \partial_{\underline{x}}\left(\frac{1+2 r^{2}+\underline{q}}{4 r^{2}+1}\right) \Lambda(\underline{q}) d x_{1} d x_{2} d x_{3}=0 .
$$

Since the integrand is infinitely differentiable and $\Lambda$ is locally integrable, and

$$
\partial_{\underline{x}}\left(H\left(\varepsilon^{2}-r^{2}\right)\right)=-2 \underline{q} \frac{\delta(\varepsilon-r)}{\varepsilon+r},
$$

we have

$$
\begin{aligned}
& \lim _{\varepsilon \rightarrow 0} \int_{0}^{\infty} \int_{S^{2}} \frac{\delta(\varepsilon-r)}{\varepsilon+r} \frac{2 r^{2}-2 \underline{x}\left(1+r^{2}\right)}{4 r^{2}+1} r^{2} \Lambda(r, \underline{\omega}) d r d S(\underline{\omega}) \\
& \quad=\lim _{\varepsilon \rightarrow 0} \int_{S^{2}} \frac{2 \varepsilon^{2}-2 \varepsilon \underline{\omega}\left(1+r^{2}\right)}{4 r^{2}+1} r^{2} \Lambda(r, \underline{\omega}) d S(\underline{\omega})=\lim _{\varepsilon \rightarrow 0}(\varepsilon-\underline{\omega}) \varepsilon^{2} \Lambda(r, \underline{\omega}) ;
\end{aligned}
$$

but

So

$$
\Lambda(r, \underline{\omega})=\frac{-2}{A_{4}} \int_{0}^{\infty} \frac{\varepsilon^{2}+t-\varepsilon \underline{\omega}}{\left(\varepsilon^{2}+t\right)^{2}+\varepsilon^{2}} d t
$$

$$
\begin{aligned}
\lim _{\varepsilon \rightarrow 0}(\varepsilon-\underline{\omega}) \varepsilon^{2} \Lambda(r, \underline{\omega}) & =-2 \frac{A_{3}}{A_{4}} \lim _{\varepsilon \rightarrow 0}(\varepsilon-\underline{\omega}) \int_{0}^{\infty} \frac{t-\underline{\omega}}{\left(1+t^{2}\right)^{2}} d t \\
& =\frac{2 A_{3}}{A_{4}} \int_{0}^{\infty} \frac{1}{\left(1+t^{2}\right)^{2}} d t=1 .
\end{aligned}
$$

Thus we have obtained the delta distribution, as stated.

Let us now show how to use Theorem 3.9 to recover Bony's formula which corresponds to the right hand side of the formula in the next result. 
Corollary 3.11. The delta distribution can be written as

$$
\begin{aligned}
\delta(\underline{q}) & =\left[-i \frac{1}{(2 \pi)^{3}} \int_{S^{2}} \frac{1+i \underline{\omega}}{\left(\langle\underline{q}, \underline{\omega}\rangle+i a|\underline{q}|^{2}\right)^{3}} d S(\underline{\omega})(1-a \underline{q})\right]_{0} \\
& =-i \frac{1}{(2 \pi)^{3}} \int_{S^{2}} \frac{1+i a\langle\underline{q}, \underline{\omega}\rangle}{\left(\langle\underline{q}, \underline{\omega}\rangle+i a|\underline{q}|^{2}\right)^{3}} d S(\underline{\omega}) .
\end{aligned}
$$

Proof. Consider $x_{0}>0$ and substitute the expression (3.4) in formula (3.7). Simple computations give the result.

Lemma 3.12. Let $f\left(q_{0}+\underline{q}\right)$ be a regular function. If the function

$$
\underline{q} \mapsto f\left(a|\underline{q}|^{2}+\underline{q}\right) \quad \text { in } \widetilde{\mathbb{H}} \backslash\{0\}
$$

has a distributional extension to $\mathbb{H}$ then the function

$$
\underline{q} \mapsto\left(\partial_{q_{0}} f\right)\left(a|\underline{q}|^{2}+\underline{q}\right) \quad \text { in } \widetilde{\mathbb{H}} \backslash\{0\}
$$

has a canonical distributional extension to $\mathbb{H}$.

Proof. Consider the paraboloid $P$ defined by $x_{0}=a|\underline{q}|^{2}$ and let $v_{1}:=v_{01}+\underline{v}_{1}$, $\underline{v}_{2}, \underline{v}_{3}$, be a basis for the tangent space at a point of the paraboloid. There exist smooth functions $A\left(|\underline{q}|^{2}\right), B\left(|\underline{q}|^{2}\right), C\left(|\underline{q}|^{2}\right)$ such that

$$
\partial_{x_{0}}+A\left(|\underline{q}|^{2}\right) v_{1}\left\langle v_{1}, \nabla_{x_{0}, \underline{q}}\right\rangle+B\left(|\underline{q}|^{2}\right)\left(\underline{v}_{2}\left\langle\underline{v}_{2}, \partial_{\underline{q}}\right\rangle+\underline{v}_{3}\left\langle\underline{v}_{3}, \partial_{\underline{q}}\right\rangle\right)=C\left(|\underline{q}|^{2}\right)\left(\partial_{x_{0}}+\partial_{\underline{q}}\right) .
$$

Denoting by $Q\left(\partial_{\underline{q}}\right)$ the operator $A \underline{v}_{1}\left\langle\underline{v}_{1}, \nabla_{x_{0}, \underline{q}}\right\rangle+B\left(\underline{v}_{2}\left\langle\underline{v}_{2}, \partial_{\underline{q}}\right\rangle+\underline{v}_{3}\left\langle\underline{v}_{3}, \partial_{\underline{q}}\right\rangle\right)$, which acts on $\left.f\left(x_{0}+\underline{q}\right)\right|_{P}=f\left(a|\underline{q}|^{2}+\underline{q}\right)$, we can write

$$
\left.\partial_{x_{0}} f\left(x_{0}+\underline{q}\right)\right|_{P}=Q\left(\partial_{\underline{q}}\right) f\left(a|\underline{q}|^{2}+\underline{q}\right) \text {. }
$$

Thus if $\tilde{f}$ is the distributional extension of $f\left(a|\underline{q}|^{2}+\underline{q}\right)$ then $Q\left(\partial_{\underline{q}}\right) \tilde{f}$ is the extension of $\left.\partial_{x_{0}} f\left(x_{0}+\underline{q}\right)\right|_{P}$.

Theorem 3.13. Let $f\left(q_{0}+\underline{q}\right)$ be a regular function. Suppose that $f\left(q_{0}+\underline{q}\right)$ locally satisfies, for $q_{0}>0$, the estimate

$$
\left|f\left(q_{0}+\underline{q}\right)\right| \leq C\left|q_{0}\right|^{-k},
$$

where $C$ is a positive constant and $k \in \mathbb{N}$. Then the function

$$
\underline{q} \mapsto f\left(a|\underline{q}|^{2}+\underline{q}\right) \quad \text { in } \widetilde{\mathbb{H}} \backslash\{0\}
$$

has a distributional extension to $\mathbb{H}$.

Proof. This is an immediate consequence of [1, 26.2]. 
Example 3.14. As an application of Theorem 3.13, we can construct the following distributions.

1. The following expression and its scalar part are distributions:

$$
\frac{1}{\pi^{2}} \frac{a|\underline{q}|^{2}-\underline{q}}{\left.|a| \underline{q}\right|^{2}-\left.\underline{q}\right|^{4}}(1-a \underline{q}) .
$$

2. The following expression and its scalar part are distributions:

$$
\frac{(1+i \underline{\omega})(1-a \underline{q})}{(\langle\underline{q}, \underline{\omega}\rangle+i a|\underline{q}|)^{3}} .
$$

\section{$\S 4$. The delta distribution in $m$ dimensions using Clifford analysis}

In this section we will show how to generalize the discussion of the previous sections to the $m$-dimensional case. We will use the setting of Clifford analysis. We will work in a Clifford algebra $\mathbb{R}_{m}$ over $m \geq 2$ imaginary units $e_{1}, \ldots, e_{m}$ satisfying the standard defining relations $e_{i} e_{j}+e_{j} e_{i}=-2 \delta_{i j}$. An element in the Clifford algebra will be denoted by $\sum_{A} e_{A} x_{A}$ where $A=i_{1} \ldots i_{r}, i_{\ell} \in\{1, \ldots, m\}, i_{1}<\cdots<i_{r}$, is a multi-index, $e_{A}=e_{i_{1}} \ldots e_{i_{r}}$ and $e_{\emptyset}=1$. As is well known, for $m>2$, the Clifford algebras $\mathbb{R}_{m}$ have zero divisors.

In the Clifford algebra $\mathbb{R}_{m}$, we can identify some specific elements with the vectors in the Euclidean space $\mathbb{R}^{m}$ : an element $\left(x_{1}, \ldots, x_{m}\right) \in \mathbb{R}^{m}$ can be identified with a so called 1-vector in the Clifford algebra through the map $\left(x_{1}, \ldots, x_{m}\right) \mapsto$ $\underline{x}=x_{1} e_{1}+\cdots+x_{m} e_{m}$.

An element $\left(x_{0}, x_{1}, \ldots, x_{m}\right) \in \mathbb{R}^{m+1}$ will be identified with the element

$$
x=x_{0}+\underline{x}=x_{0}+\sum_{j=1}^{m} x_{j} e_{j},
$$

called a paravector. Given an element $a \in \mathbb{R}_{m}$, its scalar part will be denoted by $[a]_{0}$.

The standard Radon decomposition already given in the three-dimensional case is described in the following result whose statement can also be found in [13, Theorem 1]. Below, $A_{m+1}$ will denote the surface area of the sphere of unit 1 -vectors in $\mathbb{R}^{m+1}$.

Theorem 4.1. Let $S^{m-1}$ be the sphere of unit 1-vectors. Then for $m$ even and $x_{0} \neq 0$,

$$
\begin{aligned}
& \frac{1}{A_{m+1}} \frac{x_{0}-\underline{x}}{\left|x_{0}+\underline{x}\right|^{m+1}} \\
& \quad=\operatorname{sgn}\left(x_{0}\right)(-1)^{m / 2}(m-1) ! \frac{1}{2(2 \pi)^{m}} \int_{S^{m-1}}\left(\langle\underline{x}, \underline{\omega}\rangle-x_{0} \underline{\omega}\right)^{-m} d S(\underline{\omega}),
\end{aligned}
$$


and for $m$ odd and $x_{0} \neq 0$,

$$
\begin{aligned}
& \frac{1}{A_{m+1}} \frac{x_{0}-\underline{x}}{\left|x_{0}+\underline{x}\right|^{m+1}} \\
& \quad=(-1)^{m+1 / 2}(m-1) ! \frac{1}{2(2 \pi)^{m}} \int_{S^{m-1}}\left(\langle\underline{x}, \underline{\omega}\rangle-x_{0} \underline{\omega}\right)^{-m} \underline{\omega} d S(\underline{\omega}) .
\end{aligned}
$$

Proof. The proof (see also Theorem 3.1) is based on the fact that both sides are axially monogenic, thus it is sufficient to verify the statement for $\underline{x}=0$.

Theorem 4.2. For $m$ even we have

$$
\delta(\underline{x})=(-1)^{m / 2} \frac{(m-1) !}{(2 \pi)^{m}} \int_{S^{m-1}} \operatorname{PV}\left[\langle\underline{x}, \underline{\omega}\rangle^{-m}\right] d S(\underline{\omega}) .
$$

For $m$ odd we have

$$
\delta(\underline{x})=(-1)^{(m+1) / 2} \frac{(m-1) !}{2(2 \pi)^{m}} \int_{S^{m-1}} \operatorname{BV}\left[\left(\langle\underline{x}, \underline{\omega}\rangle-x_{0} \underline{\omega}\right)^{-m}\right] d S(\underline{\omega}) .
$$

Proof. It is sufficient to take the limit as $x_{0} \rightarrow 0^{+}$in the scalar part of (4.1) and (4.2) and to recall the well known formula $\delta^{+}(\underline{x})=\frac{1}{2} \delta(\underline{x})-\frac{1}{A_{m+1}} \frac{\underline{x}}{|\underline{x}|^{m+1}}$, the scalar part being $\frac{1}{2} \delta$.

Remark 4.3. Observe that

$$
\delta(x)=\mathrm{BV}\left[\frac{-1}{2 \pi i z}\right]
$$

so

$$
\delta^{(m-1)}(x)=\mathrm{BV}\left[\frac{(-1)^{m}(m-1) !}{2 \pi i z^{m}}\right]
$$

we now replace

$$
x \mapsto\langle\underline{x}, \underline{\omega}\rangle, \quad y \mapsto x_{0}, \quad i \mapsto-\underline{\omega},
$$

to get

$$
\delta(\underline{x})=\frac{(-1)^{(m-1) / 2}}{2(2 \pi)^{m-1}} \int_{S^{m-1}} \delta_{\mathbb{R}}^{(m-1)}(\langle\underline{x}, \underline{\omega}\rangle) d S(\underline{\omega})
$$

where $\delta(\underline{x})=\prod_{\ell=1}^{m} \delta\left(x_{\ell}\right)$.

We now prove the following result:

Lemma 4.4. For $x_{0}>0$ we have

$$
\frac{1}{A_{m+1}} \frac{x_{0}-\underline{x}}{\left|x_{0}-\underline{x}\right|^{m+1}}=\frac{i^{m}(m-1) !}{2(2 \pi)^{m}} \int_{S^{m-1}} \frac{1+i \underline{\omega}}{\left(\langle\underline{x}, \underline{\omega}\rangle+i x_{0}\right)^{m}} d S(\underline{\omega}) .
$$


Proof. We limit ourselves to the case of $m$ even; the case of $m$ odd can be treated as in the proof of Proposition 3.2. Since $x_{0}>0$ we have

$$
\frac{1}{A_{m+1}} \frac{x_{0}-\underline{x}}{\left|x_{0}-\underline{x}\right|^{m+1}}=\frac{i^{m}(m-1) !}{2(2 \pi)^{m}} \int_{S^{m-1}} \frac{1}{\left(\langle\underline{x}, \underline{\omega}\rangle-x_{0} \underline{\omega}\right)^{m}} d S(\underline{\omega}) .
$$

Set

and observe that

$$
\Omega_{ \pm}:=\frac{1}{2}(1 \pm i \underline{\omega}), \quad \underline{\omega} \in S^{m-1},
$$

$$
1=\Omega_{+}+\Omega_{-}, \quad \underline{\omega} \Omega_{ \pm}=\mp i \Omega_{ \pm} .
$$

Reasoning as in Proposition 3.2, we have

$$
\frac{\Omega_{+}}{\left(\langle\underline{x}, \underline{\omega}\rangle-x_{0} \underline{\omega}\right)^{m}}=\frac{\Omega_{+}\left(\langle\underline{x}, \underline{\omega}\rangle+x_{0} \underline{\omega}\right)^{m}}{\left(\langle\underline{\omega}, \underline{\omega}\rangle^{2}+x_{0}^{2}\right)^{m}}=\frac{\left(\langle\underline{x}, \underline{\omega}\rangle-i x_{0}\right)^{m} \Omega_{+}}{\left(\langle\underline{x}, \underline{\omega}\rangle^{2}+x_{0}^{2}\right)^{m}}=\frac{\Omega_{+}}{\left(\langle\underline{x}, \underline{\omega}\rangle+i x_{0}\right)^{m}},
$$

and similarly

So we have

$$
\frac{\Omega_{-}}{\left(\langle\underline{x}, \underline{\omega}\rangle-x_{0} \underline{\omega}\right)^{m}}=\frac{\Omega_{-}}{\left(\langle\underline{x}, \underline{\omega}\rangle-i x_{0}\right)^{m}}
$$

$$
\begin{aligned}
\frac{1}{A_{m+1}} \frac{x_{0}-\underline{x}}{\left|x_{0}-\underline{x}\right|^{m+1}}= & \frac{(-1)^{m / 2}(m-1) !}{2(2 \pi)^{m}} \int_{S^{m-1}} \frac{\Omega_{+}}{\left(\langle\underline{x}, \underline{\omega}\rangle+i x_{0}\right)^{m}} d S(\underline{\omega}) \\
& +\frac{(-1)^{m / 2}(m-1) !}{2(2 \pi)^{m}} \int_{S^{m-1}} \frac{\Omega_{-}}{\left(\langle\underline{x}, \underline{\omega}\rangle-i x_{0}\right)^{m}} d S(\underline{\omega}),
\end{aligned}
$$

from which the desired equality follows, since the second summand equals the first.

Remark 4.5. This result leads to the expansion

$$
\delta(\underline{x})=\frac{i^{m}(m-1) !}{(2 \pi)^{m}} \int_{S^{m-1}} \frac{1}{(\langle\underline{x}, \underline{\omega}\rangle+i 0)^{m}} d S(\underline{\omega}) .
$$

Note that the singular spectrum of

$$
\frac{1}{(\langle\underline{x}, \underline{\omega}\rangle+i 0)^{m}}
$$

is $\{\underline{x}+i \underline{\omega}:\langle\underline{x}, \underline{\omega}\rangle=0\}$.

Remark 4.6. We have

$$
\left(\langle\underline{x}, \underline{\omega}\rangle+i 0^{+}\right)^{-m}=\left.\lim _{\underline{y} \rightarrow 0} \mathrm{BV}(\langle\underline{x}+i \underline{y}, \underline{\omega}\rangle)^{-m}\right|_{\langle\underline{y}, \underline{\omega}\rangle>0},
$$

and so the singular spectrum of $\left(\langle\underline{x}, \underline{\omega}\rangle+i 0^{+}\right)^{-m}$ is $\{\langle\underline{x}, \underline{\omega}\rangle=0\}+i \underline{\omega}$.

Our main result, whose proof mimics the proof of Theorem 3.9, is: 
Theorem 4.7. Let $x_{0}=a|\underline{x}|^{2}$. Then

$$
\delta(\underline{x})=\frac{2}{(2 \pi)^{m}}\left[\frac{a|\underline{x}|^{2}-\underline{x}}{\left.|a| \underline{x}\right|^{2}+\left.\underline{x}\right|^{m+1}}(1-a \underline{x})\right]_{0} .
$$

We now have:

Theorem 4.8. Let $f\left(x_{0}+\underline{x}\right)$ be a monogenic function for $x_{0}>0$ satisfying locally the inequality

$$
\left|f\left(x_{0}+\underline{x}\right)\right| \leq C\left|x_{0}\right|^{-k}
$$

Then

$$
\left.f\left(x_{0}+\underline{x}\right)\right|_{x_{0}=a|\underline{x}|^{2}}=\left.\lim _{\varepsilon \rightarrow 0^{+}} f\left(x_{0}+\varepsilon+\underline{x}\right)\right|_{x_{0}=a|\underline{x}|^{2}} .
$$

Proof. This is an immediate consequence of $[1,26.2]$.

As we already proved in Section 3, if we combine Lemma 4.4 and Theorem 4.7 we obtain Bony's decomposition:

Corollary 4.9. The delta distribution can be written as

$$
\begin{aligned}
\delta(\underline{x}) & =\left[\frac{i^{m}(m-1) !}{(2 \pi)^{m}} \int_{S^{m-1}} \frac{1+i \underline{\omega}}{\left(\langle\underline{x}, \underline{\omega}\rangle+i a|\underline{x}|^{2}\right)^{m}} d S(\underline{\omega})(1-a \underline{x})\right]_{0} \\
& =\frac{i^{m}(m-1) !}{(2 \pi)^{m}} \int_{S^{m-1}} \frac{1+i a\langle\underline{x}, \underline{\omega}\rangle}{\left(\langle\underline{x}, \underline{\omega}\rangle+i a|\underline{x}|^{2}\right)^{m}} d S(\underline{\omega}) .
\end{aligned}
$$

Corollary 4.10. The following equality holds in the distributional sense:

$$
\frac{1+i a\langle\underline{x}, \underline{\omega}\rangle}{\left(\langle\underline{x}, \underline{\omega}\rangle+i a|\underline{x}|^{2}\right)^{m}}=\frac{1+i a\langle\underline{x}, \underline{\omega}\rangle}{\left(\langle\underline{x}, \underline{\omega}\rangle+i\left(a|\underline{x}|^{2}+0\right)\right)^{m}} .
$$

Remark 4.11. The singular spectrum for $a=1$ is $\{0\} \times \underline{\omega}$ and if we complexify and write $\underline{x}=\underline{x}+i \underline{y}$ then $\langle\underline{x}, \underline{\omega}\rangle+i|\underline{x}|^{2}$ becomes $\langle\underline{x}, \underline{\omega}\rangle-2\langle\underline{x}, \underline{y}\rangle+i\left(\langle\underline{y}, \underline{\omega}\rangle+|\underline{x}|^{2}-\right.$ $\left.|\underline{y}|^{2}\right)$. If we set the imaginary part $\langle\underline{y}, \underline{\omega}\rangle+|\underline{x}|^{2}-|\underline{y}|^{2}>0$ we obtain a region $G$ containing $\mathbb{R}^{m}$. Hence

$$
\frac{1+i a\langle\underline{x}, \underline{\omega}\rangle}{\left(\langle\underline{x}, \underline{\omega}\rangle+i a|\underline{x}|^{2}\right)^{m}}=\lim _{\underline{y} \rightarrow 0, \underline{z} \in G} \frac{1+i a\langle\underline{z}, \underline{\omega}\rangle}{\left(\langle\underline{z}, \underline{\omega}\rangle+i a|\underline{\underline{z}}|^{2}\right)^{m}} .
$$

\section{Acknowledgements}

The first three authors are grateful to Chapman University for support and kind hospitality in the period when this paper was being written. 


\section{References}

[1] F. Brackx, R. Delanghe and F. Sommen, Clifford analysis, Pitman Res. Notes in Math. Ser. 76, 1982. Zbl 0529.30001 MR 0697564

[2] J. Bros and D. Iagolnitzer, Causality and local analyticity: mathematical study, Ann. Inst. Henri Poincaré 18 (1973), 147-184. Zbl 0286.42016 MR 0334726

[3] F. Colombo, I. Sabadini, F. Sommen and D. C. Struppa, Analysis of Dirac systems and computational algebra, Progr. Math. Phys. 39, Birkhäuser, Boston, 2004. Zbl 1064.30049 MR 2089988

[4] R. Delanghe, F. Sommen and V. Souček, Clifford algebra and spinor-valued functions, Math. Appl. 53, Kluwer, Dordrecht, 1992. Zbl 0747.53001 MR 1169463

[5] A. Fabiano, G. Gentili and D. C. Struppa, Sheaves of quaternionic hyperfunctions and microfunctions, Complex Var. 24 (1994), 161-184. Zbl 0819.30030 MR 1270307

[6] G. B. Folland, Harmonic analysis in phase space, Princeton Univ. Press, Princeton, 1989. Zbl 0682.43001 MR 0983366

[7] I. M. Gelfand and G. E. Shilov, Generalized functions I. Properties and operations, Academic Press, New York 1964. MR 0166596

[8] F. John, Plane waves and spherical means applied to partial differential equations, Interscience, New York, 1955. Zbl 0067.32101 MR 0075429

[9] A. Kaneko, Introduction to hyperfunctions, Math. Appl. (Japan. Ser.) 3, Kluwer, 1988. Zbl 0687.46027 MR 1026013

[10] I. Sabadini and D. C. Struppa, Topologies on quaternionic hyperfunctions and duality theorems, Complex Var. 30 (1996), 19-34. Zbl 0855.30039 MR 1395228

[11] F. Sommen, Hyperfunctions with values in a Clifford algebra, Simon Stevin 57 (1983), 225-254. Zbl 1054.30050 MR 0736103

[12] Microfunctions with values in a Clifford algebra II, Sci. Papers College Arts Sci. Univ. Tokyo 36 (1986), 15-37. Zbl 0649.30040 MR 0868638

[13] Clifford analysis and integral geometry, in Clifford algebra and their applications in mathematical physics, A. Micali et al. (eds.), Kluwer, 1992, 293-311. Zbl 0765.53073 MR 1199606

[14] A. Sudbery, Quaternionic analysis, Math. Proc. Cambridge Philos. Soc. 85 (1979), 199-225. Zbl 0399.30038 MR 0516081 